\title{
ATENÇÃO À CRIANÇA COM DOENÇA CRÔNICA NA ESTRATÉGIA SAÚDE DA FAMÍLIA
}

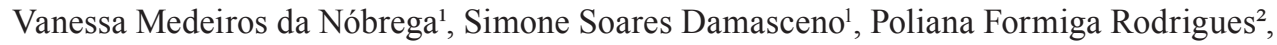
Altamira Pereira da Silva Reichert ${ }^{3}$, Neusa Collet ${ }^{4}$

\begin{abstract}
RESUMO: O aumento significativo de crianças com doenças crônicas revela a importância da qualificação dos serviços de atenção primária à saúde e de seus profissionais, no sentido de promover competências na assistência, condizentes às necessidades desta condição. Este estudo descritivo, com abordagem qualitativa, objetivou analisar a atenção à criança com doença crônica em Estratégia Saúde da Família. As entrevistas, utilizando um roteiro semiestruturado, foram realizadas no período de novembro de 2008 a janeiro de 2009 com três mães de crianças com doenças crônicas. Os dados foram analisados segundo os princípios da análise temática e emergiram duas temáticas: Não resolutividade das demandas de saúde e Insatisfação com o atendimento na Estratégia Saúde da Familia. Revelou-se a fragilidade da Estratégia para atender, com competência, às demandas de crianças com doença crônica e a necessidade de mudanças no processo de trabalho para que se efetive a assistência integral.

DESCRITORES: Atenção primária à saúde; Doença crônica; Saúde da criança.

\section{CARE FOR THE CHILD WITH A CHRONIC ILLNESS IN THE FAMILY HEALTH STRATEGY}

ABSTRACT: The significant increase in children with chronic illnesses shows the importance of the qualification of the primary health care services and of the professionals who work there, so as to promote competencies in care, befitting these conditions' needs. This descriptive study, with a qualitative approach, aimed to analyze care for the child with a chronic illness in the Family Health Strategy. The interview, using a semi-structured script, was held care in the period between November 2008 to January 2009 with three mothers of children with chronic illnesses. The data was analyzed according to the principles of thematic analysis, and two issues emerged: Failure to resolve the health needs and Dissatisfaction with the attendance in the Family Health Strategy. The study showed the Strategy's fragility in meeting - with competency - the needs of the children with chronic illnesses, and the need for changes in the work process, such that integral care may be implemented.

DESCRIPTORS: Primary health care; Chronic illness; Child health.

\section{ATENCIÓN AL NIÑO CON ENFERMEDAD CRÓNICA EN LA ESTRATEGIA SALUD DE LA FAMILIA}

RESUMEN: El aumento significativo de niños con enfermedades crónicas revela la importancia de la cualificación de los servicios de atención primaria a la salud y de sus profesionales, con fines de promover competencias en la asistencia, correspondiente con las necesidades de esta condición. Este estudio descriptivo, con abordaje cualitativo, tuvo la finalidad de analizar la atención al niño con enfermedad crónica en Estrategia Salud de la Familia. La entrevista, utilizando un guión semiestructurado, fue realizada en el periodo de noviembre de 2008 a enero de 2009 con tres madres de niños con enfermedades crónicas. Los datos fueron analizados de acuerdo a los principios del análisis temático, resultando en dos temáticas: No resolutividad de las demandas de salud y Insatisfacción con el atendimiento en la Estrategia Salud de la Familia. Se reveló la fragilidad de la Estrategia para atender, con competencia, a las demandas de niños con enfermedad crónica y la necesidad de cambios en el proceso de trabajo para que se efective la asistencia integral.

DESCRIPTORES: Atención primaria a la salud; Enfermedad crónica; Salud del niño.

${ }^{1}$ Enfermeira. Mestranda pelo Programa de Pós-Graduação em Enfermagem da Universidade Federal da Paraíba - UFPB. Membro do Grupo de Estudos e Pesquisa em Saúde da Criança e do Adolescente.

${ }^{2}$ Enfermeira. Mestranda pelo Programa de Pós-Graduação em Enfermagem da UFPB. Membro do Grupo de Estudos e Pesquisa em Saúde da Criança e do Adolescente.

${ }^{3}$ Enfermeira. Doutora em Saúde da Criança e do Adolescente. Professora do Curso de Graduação e do Programa de Pós-Graduação em Enfermagem da UFPB. Vice coordenadora do Grupo de Estudos e Pesquisa em saúde da Criança e do Adolescente.

${ }^{4}$ Enfermeira. Doutora em Enfermagem. Professora do Curso de Graduação e do Programa de Pós-Graduação em Enfermagem da UFPB. Coordenadora do Grupo de Estudos e Pesquisa em Saúde da Criança e do Adolescente.

Autor correspondente:

Recebido: $16 / 08 / 2012$

Vanessa Medeiros da Nóbrega

Aprovado: 30/01/2013

Universidade Federal da Paraíba

R. Prof. ${ }^{\text {a }}{ }^{\text {a }}$ Pinheiro de Almeida, 20 - 58050-040 - João Pessoa-PB-Brasil

E-mail: nessanobregam@hotmail.com 


\section{INTRODUÇÃO}

Na perspectiva da Atenção Primária à Saúde (APS), a atenção à saúde da criança dirige-se para o contexto da integralidade do cuidado e a Estratégia Saúde da Família (ESF) apresenta-se fundamental para vigilância da saúde da criança, acolhendo-a, inclusive, frente à doença crônica, com ações de prevenção e promoção, acompanhando e referenciando, quando necessário.

No Brasil, segundo o Instituto Brasileiro de Geografia e Estatística - IBGE, na Pesquisa Nacional por Amostra em Domicílios (PNAD) realizada em 2008, 9,1\% de crianças de 0 a 5 anos, 9,7\% de 6 a 13 anos e $11 \%$ de adolescentes de 14 a 19 anos do total geral da população nessa faixa etária tem doença crônica ${ }^{(1)}$. Esses dados são representativos e significativos para a saúde pública brasileira, exigindo olhar cuidadoso a essa população.

Concernente a essa realidade, emerge a necessidade de os profissionais de saúde estarem preparados para assistir às demandas advindas da doença crônica, acolhendo a criança e sua família em todo o processo, desde os momentos iniciais dos sinais e sintomas, até as recidivas, com cuidado holístico, contínuo, efetivo e resolutivo. Confirmando a assertiva, estudo ${ }^{(2)}$ relata que a efetiva ação da atenção primária envolve o controle e acompanhamento de doenças crônicas.

A ESF, principal proposta de reordenação do modelo assistencial em saúde no Brasil ${ }^{(3)}$ é considerada porta de entrada do sistema e, por isso, o primeiro contato dos usuários acontece nesse nível de atenção. É o espaço preferencial para promoção da saúde infantil no âmbito sanitário, considerando que a maior parte das intervenções preventivas e curativas na infância é tecnologicamente simples, dispensando cuidados hospitalares. Em contrapartida, nos casos em que são necessárias intervenções tecnologicamente mais complexas, a ESF faz a ponte entre os distintos níveis, mantendo a continuidade do cuidado por meio da coordenação da atenção ${ }^{(4)}$. Assim, funcionando adequadamente em consonância aos princípios do SUS, a ESF é capaz de ofertar uma atenção ampliada à criança com doença crônica.

Entretanto, observa-se nas práticas de saúde incoerência entre a oferta de serviços e a necessidade de atenção, não conseguindo acompanhar a tendência atual de declínio dos problemas agudos e ascensão das condições crônicas dos usuários dos serviços de saúde ${ }^{(5)}$.

No que tange à organização dos serviços de saúde, deve-se considerar que a APS só existirá como estratégia se ela cumprir suas três funções essenciais: a resolutividade, a comunicação e a responsabilização. A função de resolubilidade, inerente ao nível de cuidados primários, significa que ela deve ser resolutiva, capacitada, portanto, cognitiva e tecnologicamente para atender a mais de $85 \%$ dos problemas de sua população. A função de comunicação expressa o exercício, pela APS, ou seja, ter condições de ordenar os fluxos e contrafluxos das pessoas, dos produtos e das informações entre os diferentes componentes das redes. A função de responsabilização implica o conhecimento e o relacionamento íntimo da população adscrita nos microterritórios sanitários, o exercício da gestão de base populacional e a responsabilização econômica e sanitária em relação a essa população adscrita ${ }^{(6)}$.

Diante do aumento significativo de crianças com doenças crônicas, percebe-se a necessidade de qualificação dos serviços de APS e de seus profissionais, no sentido de promover competências na assistência condizentes às demandas singulares que emergem da condição crônica. Por isso, a avaliação contínua desses serviços é fundamental para se garantir atendimento cada vez mais resolutivo e satisfatório à criança com doença crônica e sua família. Logo, este estudo tem como objetivo analisar a atenção à criança com doença crônica em Estratégia de Saúde da Família.

\section{MÉTODO}

Trata-se de um estudo descritivo com abordagem qualitativa, realizado com mães que tiveram experiência de assistência de seus filhos com doença crônica na ESF. Foram definidos os seguintes critérios de inclusão: ser mãe de criança com doença crônica; a criança ter mais de um ano de diagnóstico de doença crônica; a família residir no perímetro urbano de João Pessoa Paraíba; e ter tido experiência anterior de atenção em ESF. Foram excluídas as pessoas que que apresentavam problemas de comunicação.

Um hospital municipal, que é referência no tratamento de criança com doença crônica, serviu inicialmente de local para a produção do material empírico, no período de novembro de 2008 a janeiro de 2009. Na pré-seleção foi realizado levantamento de dados nos prontuários, a fim de buscar informações de crianças com doença crônica residentes no município de João Pessoa. Durante o período de coleta de dados, observou-se que cerca de 90\% das crianças hospitalizadas por problemas crônicos residiam em outros municípios. A partir destes dados, as pesquisadoras aproximaram-se do acompanhante das crianças, a fim de verificar se o mesmo atendia 
aos critérios de inclusão. Confirmada a elegibilidade, o convite para participação da pesquisa era realizado com a devida apresentação do objetivo do estudo. Ao aceitar, o responsável pela criança formalizava a participação e agendava-se a entrevista, de acordo com a sua disponibilidade de tempo. Portanto, foram eleitas para participação neste estudo, conforme os critérios esclarecidos, três mães de crianças com doença crônica.

Para coleta de dados utilizou-se um roteiro de entrevista semiestruturado, constituído por duas partes: a primeira, com dados da criança (idade, sexo, tipo e tempo de diagnóstico da doença crônica); e a segunda com a questão norteadora: "Fale-me sobre a atenção na ESF a seu filho com doença crônica, desde o início dos primeiros sinais e sintomas da doença, até os dias atuais".

As entrevistas, com duração média de 30 minutos, foram gravadas e transcritas na íntegra. Os dados processados a partir dos princípios da análise temática contemplaram a pré-análise, que corresponde ao contato inicial com o material coletado, utilizando a leitura flutuante e determinando as unidades de registro ou palavras-chave; exploração do material, com a realização dos recortes do texto, classificação e agregação dos dados; e, por fim, o tratamento dos resultados obtidos e interpretação ${ }^{(7)}$.

A produção do material empírico iniciou-se após aprovação do projeto pelo Comitê de Ética em Pesquisa do hospital em que se realizou o estudo, sob o protocolo n. 090/08, e foi norteada pelas diretrizes e normas regulamentadoras de pesquisa envolvendo seres humanos estabelecidas pela Resolução 196/96 do Conselho Nacional de Saúde ${ }^{(8)}$. Como garantia do anonimato, as mães participantes do estudo foram identificadas pela letra $(\mathrm{M})$ acompanhadas de números ordinais nos resultados e discussão (M1, M2 e M3), concernente à ordem de realização das entrevistas.

\section{RESULTADOS}

A faixa etária das mães entrevistadas variou entre 28 e 39 anos. No que diz respeito à escolaridade, duas possuíam o ensino fundamental completo e uma o ensino médio completo. Uma das participantes desempenhava atividade remunerada como vendedora e duas eram do lar. Com relação às crianças, o sexo masculino foi unânime, com as seguintes idades, doenças crônicas e tempo de diagnóstico: onze anos de idade, diagnosticado com Anemia Falciforme há seis anos; oito anos, com diagnóstico de Leucemia Linfóide Aguda há um ano; e sete anos, acometido por Feocromocitoma, tumor no pâncreas, com cinco anos de diagnóstico.
A partir da análise dos depoimentos das mães, foram identificados dois núcleos temáticos, sendo eles: Não resolutividade das demandas de saúde e Insatisfação com o atendimento na ESF.

\section{Temática 1: Não resolutividade das demandas de saúde}

Os resultados apontam que o relacionamento entre a equipe que atua na ESF e as mães não é sempre caracterizado por acolhimento, acessibilidade e satisfação. As falas revelam sentimentos de descrédito na capacidade resolutiva da ESF, em consequência do não atendimento às demandas apresentadas:

Não frequento o PSF, pois toda vez que preciso de uma consulta não consigo, remédio sempre não tem [...]. É uma negação. Eu nunca levo ele assim para o PSF. Só para verificar a pressão, ai se tiver alta eu venho para cá, porque lá é uma negação porque tudo lá vai para trás [...]. (M3)

O postinho [USF] é assim, quando eu preciso deles eles nunca me atendem. Às vezes eu chego do trabalho às 17 horas e ele [Filho] está com a garganta inflamada ai eu procuro a unidade, pois lá funciona até umas 19, 20 horas. Quando eu procuro não consigo atendimento, pois tem muita gente, então não consigo encaixe. (M1)

Além da dificuldade de acesso e falta de medicamentos, as mães entrevistadas referiram a falta de interesse e o despreparo dos profissionais atuantes na atenção básica:

Quando meu filho estava doente procurava sempre o serviço e a médica dizia que era virose, que era para colocar meu filho para comer. Dizia que internar não adianta. Dizia vá para casa e passava uns remédios para ele [...]. É porque é uma médica bem estilosa. Meu filho passou seis meses indo para lá e ela mandava ele para casa. (M2)

A estrutura física compartilhada por mais de uma equipe da ESF é denominada de unidades de saúde da família integradas, na qual as equipes de saúde dividem o mesmo espaço, porém, possuem distintas áreas de abrangência. No município onde ocorreu a pesquisa existem 21 unidades integradas, fato que por vezes confunde o usuário quanto a sua vinculação a determinada equipe. No relato a seguir, observa-se que a falta de interesse de um determinado profissional de saúde fez a família recorrer a outro da unidade integrada. No entanto, por não ser da sua área de abrangência, este 
não podia acompanhar a criança, demonstrando que houve escuta, porém o atendimento não foi resolutivo.

O médico da minha área é o doutor [nome do médico] ele é gastro, então nem demonstrou estar interessado em procurar saber sobre a doença crônica de meu filho [...]. A única que se interessou foi uma médica pediatra da outra unidade porque eu moro em [bairro que reside] e lá as unidades são integradas em um mesmo espaço. [...] Então fui explicar porque eu não queria mais passar em doutor [nome do médico] ai elas perguntaram: "por que mãe?" Eu expliquei, elas disseram que ele [o médico] não podia fazer isso não, tinha que procurar saber, pois era uma criança hipertensa da área dele, é uma criança que tem uma doença crônica, ele tem que procurar saber. Por isso, eu quase não vou lá. (M3)

\section{Temática 2: Insatisfação com o atendimento na ESF}

As famílias demonstraram insatisfação com o atendimento dos profissionais de saúde da ESF, repercutindo negativamente na formação de vínculo e na atenção à criança com doença crônica, como ilustra o recorte:

Não gosto [referindo-se à ESF]. É péssimo, coloque aí: tanto a médica quanto o atendimento. Se eu pudesse não tinha nenhum vínculo com o PSF. (M2)

A insatisfação deve-se ao fato de a família não sentir suas demandas de atendimento à saúde contempladas pelos profissionais, como exemplifica o depoimento:

Só o hospital mesmo! Porque aqui [ESF] não tem como, eles são muito assim [...] não estão nem aí, dizem para ir para um pronto-socorro de urgência. (M1)

Eu já levei ele [filho] na USF quando ele estava muito doentinho, a pressão dele estava alta e o médico disse que lá não resolvia o caso dele. Ai eu disse: "eu não quero que você resolva, eu quero que você adiante algo, pois eu sei o que ele tem e queria que você adiantasse para pelo menos chegar ao hospital". Ai ele disse para procurar o médico que acompanha ele nessas coisas, pois lá não resolvia. (M3)

\section{DISCUSSÃO}

Observam-se lacunas importantes no acesso de primeiro contato, um dos princípios ordenadores da atenção básica em saúde, uma vez que, o mesmo "im- plica a acessibilidade e o uso do serviço a cada novo problema ou novo episódio de um problema pelo qual as pessoas buscam atenção à saúde" ${ }^{"(9: 62)}$. Essas lacunas no acesso da família da criança compromete a formação de vínculo e a satisfação com o serviço, gerando descrença neste âmbito de atenção à saúde.

No caso de usuários com doenças crônicas, este quadro pode ser ainda mais impactante, por ser geralmente o tratamento prolongado, complexo e exigir cuidados constantes em relação à terapêutica em si e aos determinantes desencadeadores do agravamento a saúde da criança. É nesse contexto que a equipe de saúde precisa estar engajada no processo de cuidar da criança com doença crônica. Para tanto, necessita conhecer a enfermidade, suas manifestações e implicações ${ }^{(10)}$. Assim, é indispensável mudanças na postura dos profissionais, no cotidiano da atenção e na formação, para que os mesmos estejam capacitados para lidar com situações de cuidado intrínsecas à condição crônica na infância.

Em estudo ${ }^{(11)}$ foi identificado que, na tentativa de atender à demanda, a equipe acaba, muitas vezes, por limitar o acesso. Dessa forma, mantém-se o caráter excludente do acesso e, decorrente disso, observa-se descaso à saúde da população adstrita, que muitas vezes perde tempo em filas para o acesso ao atendimento, situação também observada na presente pesquisa.

Transformações no trabalho em saúde são necessárias para ampliar o acesso da população e reduzir as filas de espera. $\mathrm{O}$ acolhimento mostra-se como ferramenta indispensável à ESF, pois, ao utilizar essa estratégia, a equipe é capaz de oferecer atendimento mais resolutivo ${ }^{(12)}$. Também é imprescindível a escuta qualificada das necessidades da população, proporcionando maior satisfação do usuário.

Contrário às necessidades de saúde inerentes ao contexto atual, têm-se consultas pontuais, usualmente direcionadas à sintomatologia aguda apresentada pela criança e sequencialmente a administração de medicamentos, conhecida como atendimento queixa-conduta. As doenças crônicas usualmente são caracterizadas por sinais e sintomas comuns a outras enfermidades e, como o profissional da atenção primária à saúde tem pouca vivência em tratar crianças com doença crônica, em muitas situações não se encontram capacitados para a definição do diagnóstico precoce.

Ao realizar um diagnóstico equivocado devido à ausência de evidências, de informações advindas dos exames laboratoriais, exame físico, medidas antropométricas, histórico; o profissional não esclarece 
o problema identificado e não fornece explicações referentes à doença da criança, ao tratamento a ser seguido, às precauções para evitar a recidivas. Como consequência, o responsável pela criança considera a consulta na ESF como insatisfatória, uma vez que permanece com dúvidas e incertezas ${ }^{(13)}$.

As expectativas dos acompanhantes em relação à consulta estão centradas no esclarecimento das dúvidas acerca da situação de saúde da criança. Em alguns casos, evidencia-se a expectativa de bom relacionamento interpessoal, na forma de atenção, segurança, carinho e interesse por parte do profissional ${ }^{(13)}$. Este estudo, porém revelou não se fazerem presentes esses atributos no momento em que os pais chegam com a sua criança na ESF, confirmando a falta de resolutividade dos problemas de saúde e a insatisfação com o atendimento recebido.

Outra situação evidenciada nesse estudo foi a dificuldade enfrentada por usuários de unidades de saúde da família integradas, quando nem sempre conseguem a vinculação a determinado profissional e se vem impossibilitados de acionar outro na mesma unidade. Estudo $^{(14)}$ corrobora essa evidência ao constatar que o aproveitamento da estrutura física por mais de uma equipe pode dificultar a acessibilidade e é considerado um problema para os usuários. A conduta profissional, possivelmente condicionada pela supervisão, baseada no território de abrangência e relacionada à organização da unidade da ESF, cria obstáculos e, com isso, o usuário fica irritado, frustrado e decepcionado por não receber o atendimento desejado no momento de sua necessidade.

Essa situação revela a vulnerabilidade do sistema de saúde e de seus profissionais, nem sempre preparados para atender a complexidade do cuidado às pessoas com doença crônica de forma resolutiva, eficiente, humanizada e integral, tanto no interior dos serviços de atenção à saúde, quanto para assistir adequadamente aquelas pessoas que necessitam de continuidade de cuidados no domicílio ${ }^{(15)}$.

Na superação deste obstáculo, que inviabiliza a efetivação da integralidade na atenção primária, tem-se a longitudinalidade do cuidado, a qual corresponde a uma relação pessoal de longa duração entre os profissionais de saúde e os pacientes em suas unidades de saúde, independente da presença de doença. Ter atenção longitudinal significa que aqueles indivíduos na população identificam uma fonte de atenção como "sua" (9). É um dos atributos da atenção primária em saúde e apresenta como dimensões a existência e o reconhecimento de uma fonte regular de cuidados, o estabelecimento de vínculo terapêutico duradouro entre trabalhadores de saúde e continuidade informacional ${ }^{(16)}$. Este atributo, quando colocado em prática, se revela indispensável no seguimento dos cuidados de crianças com doenças crônicas, pois favorece atenção efetiva, e singular promovendo, também, a garantia de outro atributo da APS, a continuidade do cuidado ou uma atenção orientada para a criança ao longo do tempo ${ }^{(9)}$.

Constatou-se que a insatisfação acontece em decorrência da não resolutividade as demandas apresentadas pelas crianças com doenças crônicas, pois quando as famílias não são acolhidas, mostram-se insatisfeitas com o serviço, dificultando a construção de vínculo. $\mathrm{O}$ vínculo remete ao campo da bioética e pressupõe, em primeira instância, relação interpessoal duradoura e agradável, envolve confiança, comunicação efetiva, consideração dos problemas relatados e dos aspectos biopsicossociais do paciente ${ }^{(16)}$.

O usuário, ao procurar pelo serviço de saúde, espera que a relação com os profissionais gere acolhimento e permita resolução ou amenização das causas do seu sofrimento, ou problema, uma vez que a escuta sensível é condição primordial para a avaliação da saúde e terapêutica correta. Para haver tal vínculo, é necessária a ascensão do usuário à condição de sujeito que fala e julga, sem o qual não se estabelecem relações adequadas. Dessa forma, o estabelecimento de vínculo qualifica a consulta, por se relacionar ao atendimento de uma necessidade dos usuários ${ }^{(13)}$.

Quando tratados de forma resolutiva, os cuidadores das crianças partilharão sua realidade, fraquezas e sofrimentos com aqueles profissionais que demonstrarem, além da capacidade de escuta, desempenho clínico de acordo com as necessidades da criança e da família. Nessa perspectiva, os profissionais da equipe de saúde da família devem proporcionar atendimento clínico aos usuários com continuidade, possibilitando a criação de vínculos $^{(17)}$ e responsabilizações, determinantes para o atendimento humanizado e integral.

Ao não encontrarem garantia do acesso de primeiro contato na USF e não terem seus problemas de saúde resolvidos, as famílias de crianças com doença crônica buscam atendimento nos níveis secundários e terciários. O não estabelecimento de vínculo e confiança entre família e equipe na USF tem alimentado o imaginário popular de que a atenção em nível hospitalar resolve todos os problemas relacionados à saúde ${ }^{(14)}$. Essa cultura e modo de organização dos serviços de saúde na APS fragilizam a construção do cuidado em rede, rico e ampliado para resolutividade no nível primário de atenção à saúde. 
Ao procurar atendimento, as mães também buscam um espaço de comunicação, de diálogo, de escuta qualificada, cabendo à equipe de saúde abrir espaços que promovam a interação entre família e equipe, permitindo a expressão de experiências, sentimentos, anseios e resolução de dúvidas advindas do enfrentamento da doença crônica na infância.

Vale salientar que a situação de saúde de forte predomínio relativo às condições crônicas não pode ser respondida com eficiência, efetividade e qualidade por sistemas de saúde voltados, prioritariamente, para as condições agudas e para as agudizações de condições crônicas e organizados de forma fragmentada ${ }^{(6)}$.

Ressalta-se a significância das discussões propostas por este estudo em um dos trechos de uma das entrevistas em relação à resposta do médico, o qual afirmou que a ESF não solucionava o problema da criança. Ao analisar a atenção oferecida a essas crianças, percebe-se que a prática está fragilizada neste nível de atenção, transformando a ESF em um espaço de respostas não resolutivas às necessidades de saúde das crianças com doenças crônicas.

\section{CONSIDERAÇÕES FINAIS}

As discussões tecidas neste estudo revelam situação inquietante no que diz respeito à atenção de criança com doença crônica, pois a promoção da saúde integral da criança deve ir além da atenção com os estados agudos, englobando o compromisso mútuo entre instituições governamentais e sociedade pela sua qualidade de vida.

Apreende-se que a atenção à saúde em nível primário, porta de entrada do sistema, não está capacitada e demonstra-se fragilizada para atender de modo resolutivo às demandas de crianças com doença crônica. Identificou-se que o foco da atenção está na queixa-conduta e de modo fragmentado, intensificando a cultura de busca pelos serviços secundários e terciários de atenção à saúde, mais centrado no hospital. Assim, a USF distancia-se de um dos principais atributos da APS, sua configuração como acesso de primeiro contato, inviabilizando, inclusive, a promoção da atenção integral e ampliada, fato que gera insatisfação e descrédito na estratégia de saúde da família.

Satisfação e vínculo são anseios dos usuários exigindo escuta qualificada, aliada ao desempenho clínico dos profissionais para alcançá-los. Salienta-se, ainda, que o acolhimento deve nortear a produção do cuidado, pois esse atributo é capaz de identificar as necessidades da criança e sua família por meio da escuta e valorização da queixa trazida, aspecto também promotor de vínculo.
Os resultados encontrados neste estudo contribuem para reflexão acerca do atendimento que vem sendo ofertado às crianças com doenças crônicas na estratégia de saúde da família, fazendo-nos compreender quais mudanças são imperativas para que se efetive a assistência a esta demanda no nível primário de atenção.

Pontua-se como limitação deste estudo o pequeno número de sujeitos entrevistados, resultado da delimitação do período para coleta de dados no local selecionado. Embora seja um hospital de referência para doenças crônicas no município onde o estudo foi realizado, no período da coleta houve poucas internações de crianças que atendiam os critérios de inclusão estabelecidos. Contudo, salienta-se que os depoimentos das famílias foram abrangentes e contemplaram sentidos e significados importantes para interpretação, resultando em uma reflexão relevante acerca da atenção à criança com doença crônica na ESF. Devido aos problemas identificados nesta pesquisa, ressalta-se a relevância de novos estudos com a mesma temática a fim de aprofundar o entendimento acerca dos modos como o cuidado a essa população tem sido realizado na ESF, evidenciando ferramentas e tecnologias que proporcionem um cuidado ampliado, singular e resolutivo.

\section{AGRADECIMENTOS:}

Ao Conselho Nacional para o Desenvolvimento Científico e Tecnológico - CNPq pelo financiamento. Processo n. 476666/2007-4

\section{REFERÊNCIAS}

1. Instituto Brasileiro de Geografia e Estatística. Pesquisa Nacional por Amostra de Domicílios: um panorama da saúde no Brasil, acesso e utilização dos serviços, condições de saúde e fatores de risco e proteção à saúde, 2008. Rio de Janeiro: Fiocruz/MS/IBGE; 2010.

2. Alfradique ME, Bonolo PF, Dourado I, Lima-Costa $\mathrm{MF}$, Macinko J, Mendonça CS, et al. Internações por condições sensíveis à atenção primária: a construção da lista brasileira como ferramenta para medir o desempenho do sistema de saúde (Projeto ICSAP Brasil). Cad. Saúde Pública. 2009; 25(6):1337-49.

3. Kantorski LP, Jardim VMR, Pereira DB, Coimbra VCC, Oliveira MM. A integralidade no cotidiano de trabalho na Estratégia Saúde da Família. Rev. Gaúcha Enferm. 2009;30(4):594-601.

Cogitare Enferm. 2013 Jan/Mar; 18(1):57-63 
4. Harzheim E, Stein AT, Álvarez-Dardet C. A efetividade dos atributos da atenção primária sobre a saúde infantil. Bol saude. 2004;18(1):23-40.

5. Ministério da Saúde (BR). Secretaria de Atenção à Saúde. Departamento de Ações Programáticas e Estratégicas. Linha de cuidado para a atenção integral à saúde de crianças, adolescentes e suas famílias em situação de violências: orientação para gestores e profissionais de saúde. Brasília; 2010.

6. Mendes EV. O cuidado das condições crônicas na atenção primária à saúde: o imperativo da consolidação da estratégia saúde da família. Brasília: Organização Pan-Americana da Saúde; 2012.

7. Minayo MCS. O desafio do conhecimento: pesquisa qualitativa em saúde. 10ª ed. São Paulo: Hucitec; 2007.

8. Ministério da Saúde (BR). Conselho Nacional de Saúde. Dispõe sobre diretrizes e normas regulamentadoras de pesquisa em seres humanos. Resolução n. 196, de 10 de outubro de 1996. [Internet] Brasília; 1996. [acesso em 14 ago 2012]. Disponível: http://conselho.saude.gov.br/ resolucoes/reso_96.htm.

9. Starfield B. Atenção primária: equilíbrio entre necessidades de saúde, serviços e tecnologia. Brasília: UNESCO; 2002.

10. Araújo YB, Collet N, Moura FM, Nóbrega RD. Conhecimento da família acerca da condição crônica na infância. Texto Contexto-Enferm. 2009;18(3):498-505.

11. Schimith MD, Lima MAD. Acolhimento e vínculo em uma equipe do Programa Saúde da Família. Cad. Saúde Pública. 2004;20(6):1487-94.

12. Takemoto MLS, Silva EM. Acolhimento e transformações no processo de trabalho de enfermagem em unidades básicas de saúde de Campinas. Brasil. Cad. Saúde Pública. 2007;23(2):331-40.

13. Assis LCF, Veríssimo MLOR. Expectativas e necessidades de acompanhantes de crianças na consulta de saúde. Rev. bras. crescimento desenvolv. hum. 2010;20(2):317-29.

14. Silva LOL, Dias CA, Soares MM, Rodrigues SM. Acessibilidade ao serviço de saúde: percepções de usuários e profissionais de saúde. Cogitare enferm. 2011;16(4):654-60.

15. Almeida MI, Molina RCM, Vieira TMM, Higarashi IH, Marcon SS. O ser mãe de criança com doença crônica: realizando cuidados complexos. Esc. Anna Nery. 2006;10(1):36-46.

16. Cunha EM, Giovanella L. Longitudinalidade/ continuidade do cuidado: identificando dimensões e variáveis para a avaliação da Atenção Primária no contexto do sistema público de saúde brasileiro. Ciênc. saude colet. 2011;16(Suppl 1):1029-42.

17. Pina JC, Mello DF, Mishima SM, Lunardelo SR. Contribuições da estratégia atenção integrada as doenças prevalentes na infância ao acolhimento de crianças menores de cinco anos. Acta Paul. Enferm. 2009;22(2):142-8. 